\title{
Ethyl pyruvate can alleviate alcoholic liver disease through inhibiting Nrf2 signaling pathway
}

\author{
FEI SHEN ${ }^{1}$, ZHAOHONG WANG $^{1}$, WEI LIU ${ }^{2}$ and YUJI LIANG ${ }^{3}$ \\ ${ }^{1}$ Department of Liver Disease, Jinan Infectious Disease Hospital, Jinan, Shandong 250000; \\ ${ }^{2}$ Department of Infectious Disease, Rizhao People's Hospital, Rizhao, Shandong 276800; \\ ${ }^{3}$ Institute of Materia Medica, Shandong Academy of Medical Sciences, Jinan, Shandong 250062, P.R. China
}

Received October 13, 2017; Accepted January 19, 2018

DOI: $10.3892 /$ etm.2018.5925

\begin{abstract}
The effects of ethyl pyruvate (EP) on alcoholic liver disease and its related mechanism were investigated. Thirty male C57/BL6 mice were randomly divided to three groups: Control $(n=10)$, alcoholic liver disease (ALD, $n=10)$ and ethyl pyruvate group (EP, $n=10)$. EP group was treated with gavage using EP (100 mg/kg) for 15 consecutive days. Control and ALD group were treated with the same volume of normal saline. After the last gavage, EP and ALD group were treated with the intraperitoneal injection of $50 \%$ alcoholic solution $(10 \mathrm{ml} / \mathrm{kg})$. After that, ALD and EP group received the gavage using alcohol for 4 weeks, while Control group received the same volume of normal saline, and blood and liver tissues were taken for detection. Results showed that in this experimental study that EP could effectively alleviate the alcoholic liver disease. The levels of alanine aminotransferase (AST), triglycerides (TG), free fatty acid (FFA) and FBG in EP group were significantly lower than those in ALD group, but the number of platelets was reversed, and the differences were statistically significant; the levels of anti-inflammatory factors (TGF- $\beta /$ IL-10) and superoxide dismutase (SOD) in EP were significantly higher than those in ALP group, but the levels of pro-inflammatory factors (IL-6/TNF- $\alpha$ ) and MDA were significantly lower than those in ALP group. EP upregulated CYP2E1, downregulated PPAR- $\alpha$, nuclear factor 2 (Nrf2) and very-low density lipoprotein receptor (VLDLR), positively regulated the CYP2E1-PPAR- $\alpha$-ROS signaling pathway and negatively regulated the ROS-Nrf2-VLDLR signaling pathway. EP can increase anti-inflammatory factors and decrease proinflammatory factors, enhance the activity of SOD and reduce FFA and TG. Moreover, it can upregulate the PPAR- $\alpha$ expression by negative regulation of CYP2E1-PPAR- $\alpha$ signaling
\end{abstract}

Correspondence to: Dr Yuji Liang, Institute of Materia Medica, Shandong Academy of Medical Sciences, 22029 Jingshi Road, Jinan, Shandong 250062, P.R. China

E-mail: ouzongshao@163.com

Key words: ethyl pyruvate, alcoholic liver disease, Nrf2 pathway and downregulate the Nrf2 expression by negative regulation of Nrf2-VLDLR signaling pathway, thus alleviating the alcoholic liver disease.

\section{Introduction}

Alcoholic liver disease (ALD) refers to the liver injury caused by excessive drinking (1), the early clinical symptoms mainly include alcoholic fatty liver, alcoholic hepatitis and alcoholic liver fibrosis, and it can ultimately develop into the end-stage liver disease. With the improvement of people's living standards and the sharp increase in alcohol consumption, its morbidity and mortality rates are increasingly higher in the world (2). The specific pathogenesis of ALD is more complex, and a large number of studies have shown that the oxidative stress, inflammation and lipid metabolism disorder (3) caused by alcohol metabolism play key roles in the occurrence and development of ALD. The relevant mechanism research has shown that alcohol metabolism can increase the synthesis of triglycerides (TG) in the body. The increased TG and the accumulation of fatty acids in hepatocytes result in the fatty degeneration of liver (4), and excessive fat accumulation can also improve the oxidative stress and inflammatory response, thereby aggravating ALD and leading to lipid hepatitis (5). Some relevant studies have also confirmed that the overexpression of very-low density lipoprotein receptor (VLDLR) in hepatocytes is an important mechanism leading to alcoholic fatty liver (6). In the lipid hepatitis caused by high-fat diet, the overexpression of VLDLR in hepatocytes induced by endoplasmic reticulum stress plays an important role (7). The expression of VLDLR is regulated by nuclear factor 2 (Nrf2), and oxidative stress can activate Nrf2. Therefore, the upregulation of Nrf2 caused by oxidative stress in ALD can positively regulate the protein expression of VLDLR (6).

Ethyl pyruvate (EP) has strong anti-bacterial, anti-virus, anti-inflammatory, anti-allergy and vasodilator effects (8). Many experimental studies have also confirmed its strong anti-oxidant and anti-apoptotic effects (9). Therefore, it is boldly speculated that EP can alleviate the liver damage caused by ALD. This study aimed to investigate the protective effect of EP on ALD and its possible relevant mechanism using the ALD mouse model $(10,11)$. 


\section{Materials and methods}

Animals and treatments. A total of 30 male C57/BL6 mice of clean grade aged 6-8 weeks weighing $21 \pm 2 \mathrm{~g}$ were randomly divided to three groups: normal control group (Control group, $\mathrm{n}=10$ ), alcoholic liver disease group (ALD group, $\mathrm{n}=10$ ) and ethyl pyruvate group (EP group, $n=10$ ). EP group was pretreated with gavage using EP $(100 \mathrm{mg} / \mathrm{kg})$ for 15 consecutive days. Control group and ALD group were treated with the same volume of normal saline. After the last gavage, EP and ALD group were treated with the intraperitoneal injection of $50 \%$ alcoholic solution $(10 \mathrm{ml} / \mathrm{kg})$. This study was approved by the Animal Ethics Committee of Institute of Materia Medica of Shandong Academy of Medical Sciences (Jinan, China). After that, ALD and EP group were treated with alcohol $(4 \mathrm{~g} / \mathrm{kg} \cdot d a y$, alcohol content of 52\%); alcohol weight $(\mathrm{g})=$ liquor volume $(\mathrm{ml}) \mathrm{x}$ alcohol content in liquor $(\mathrm{v} / \mathrm{v}) \mathrm{x}$ alcohol density $(0.8 \mathrm{~g} / \mathrm{ml})$. Mice were fed for 4 weeks and mice in Control group were treated with the gavage using the same volume of normal saline. After the gavage, blood was drawn from the retrobulbar venous plexus of mice in each experimental group. Instantly, one part of it was used to measure fasting blood glucose (FBG) and to count platelets, and for statistical analysis; another part was centrifuged for $10 \mathrm{~min}$ at $2,500 \mathrm{x} \mathrm{g}$ to separate the serum. Then a small piece of liver tissue was taken from the same part of the right lobe of liver in the thoracotomy. Liver homogenate $(10 \%)$ was prepared using the normal saline at $4^{\circ} \mathrm{C}$, and centrifuged for $10 \mathrm{~min}$ at $2,500 \mathrm{x} \mathrm{g}$; and the supernatant was taken. Superoxide dismutase (SOD) and malondialdehyde (MDA) in liver tissues in each group were measured. The samples of left lobe of liver were soaked in $4 \%$ formaldehyde solution, and the remaining liver tissues were cut into small pieces and stored at $-80^{\circ} \mathrm{C}$ for detection later.

Serum aspartate aminotransferase (AST), Alanine aminotransferase (ALT), triglyceride (TG), free fatty acid (FFA) assays. Liver enzyme and blood lipid in the serum samples collected above were detected, including ALT, AST, TG and FFA. Experimental animals (Keygen, Nanjing, China) were used in the detection using the fully automatic biochemical analyzer.

Detection of morphological changes in liver tissues and apoptosis. After fixation using $4 \%$ formaldehyde solution, the liver tissues collected from each experimental group were prepared into paraffin sections according to the typical method (12). The pathological changes in liver tissues were observed under an optical microscope (x400).

RNA isolation and real-time PCR. Total RNA was isolated from frozen liver tissues and serum and real-time reverse transcription-polymerase chain reaction (RT-PCR) was performed as previously described (13). The primers used in the present study (Table I) were selected from the PubMed database. The real-time PCR data were analyzed using the relative gene expression (i.e., ${ }^{\Delta A} \mathrm{Cq}$ ) method (University of Leicester, London, UK).

SOD and MDA concentration assays. The oxidative stress indexes of the serum samples and liver tissue homogenate
Table I. Primer sequences.

\begin{tabular}{ll}
\hline Gene & \multicolumn{1}{c}{ Sequence } \\
\hline IL-6 & F: 5'-CCCTGCGTTTCTCTGCAAAC-3' \\
& R: 3'-TTTCAGGGTGGAAGGCAGAC-5' \\
TNF- $\alpha$ & F: 5'-CATCCGTTCTCTACCCAGCC-3' \\
& R: 3'-AATTCTGAGCCCGGAGTTGG-5' \\
IL-10 & F: 5'-CCTCTGGATACAGCTGCGAC-3' \\
& R: 3'-GTAGATGCCGGGTGGTTCAA-5' \\
TGF- 31 & F: 5'-AGGGCTACCATGCCAACTTC-3' \\
& R: 3'-CCACGTAGTAGACGATGGGC-5' \\
PPAR- $\alpha$ & F: 5'-GGGTACCACTACGGAGTTCACG-3' \\
& R: 5'-CAGACAGGCACTTGTGAAAACG-3' \\
CYP2E1 & F: 5'-CTTGCTTGTCTGGATCGCCA-3' \\
& R: 5'-GGGGCAGGTTCCAACTTCTA-3' \\
Nrf2 & F: 5'-GGGTACCACTACGTTCACAG-3' \\
& R: 5'-CAGACAGGCACTTGTGACG-3' \\
VLDLR & F: 5'-CTCTTGTCTGGATCAAGCCA-3' \\
& R: 5'-GGCAGGTTCCAACAATTCTA-3' \\
\hline
\end{tabular}

F, forward; R, reverse.

collected above were detected, including MDA and SOD. MDA content was detected using the thiobarbituric acid (TBA) method; and SOD activity was determined using the nitrite method (14).

Western blot analysis. Liver tissues were homogenized and proteins were detected by western blot using specific antibodies as previously described (13). Anti-CYP2E1 anti-PPAR- $\alpha$, anti-Nrf2, anti-VLDLR and anti-GAPDH were obtained from Abcam (Cambridge, UK).

Statistical analysis. SPSS 18.0 (version X; IBM, Armonk, NY, USA) software was used for the statistical analysis of relevant experimental data in this study. All data are presented as mean \pm standard deviation. The differences between the two groups were compared using t-test. $\mathrm{P}<0.05$ suggested that the difference was statistically significant.

\section{Results}

EP reduces ALT/AST levels and liver morphological changes caused by $A L D$, inhibites TG deposition and reduces FFA level. As shown in Fig. 1, both the significantly increased ALT/AST (Fig. 1A) levels and heavier fatty changes (Fig. 1E) in ALD group compared with those in Control group, proved that intraperitoneal injection of alcohol can cause acute alcoholic liver injury. The significant increases in TG/FFA (Fig. 1B) and FBG (Fig. 1C) confirmed that acute alcoholic liver injury can lead to ALD; compared to the ALD group, the significantly decrease of ALT/AST, TG/FFA, FBG in EP group coupled with the significant increase of platelet in EP (Fig. 1D), proved that EP can alleviate liver injury caused by alcohol and prevent ALD. 
A

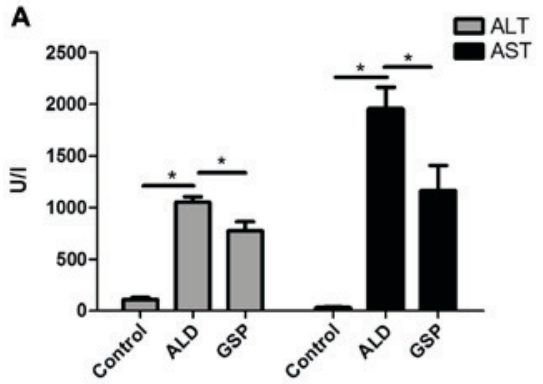

C

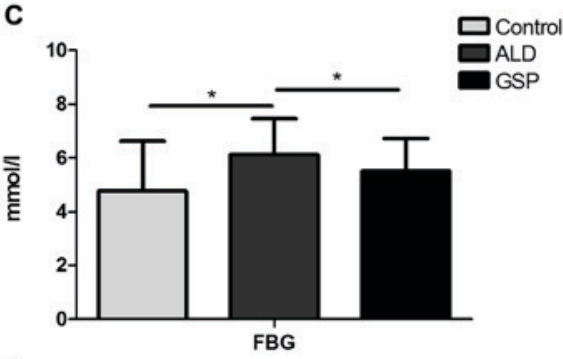

B
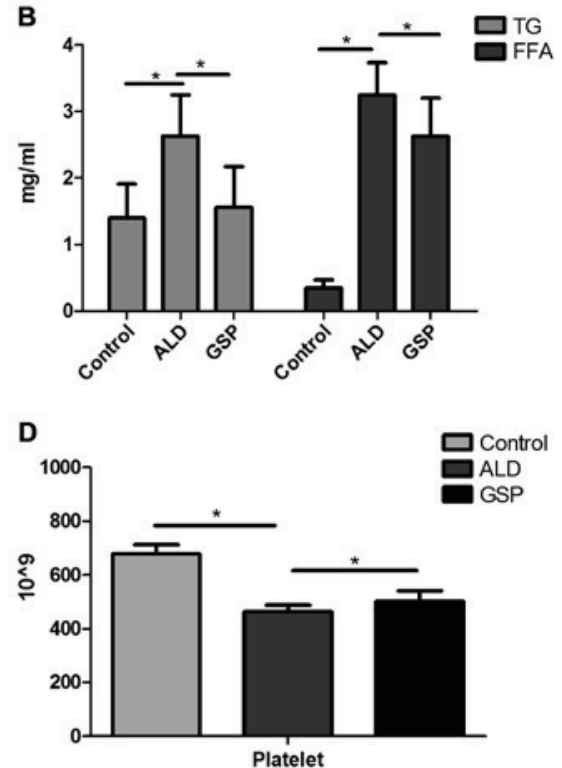

E
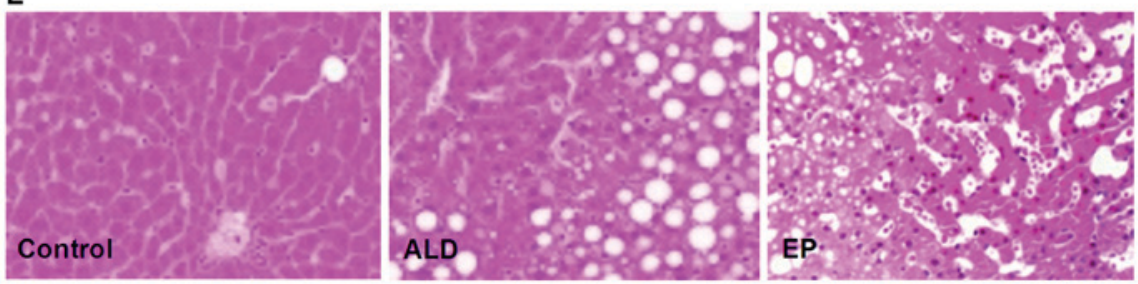

Figure 1. Comparisons of ALT/AST (A), TG/FFA (B), FBG (C) and platelet (D) levels in the serum in each experimental group ("P<0.05); and the different histological changes (E) in each experimental group. ALT, Alanine aminotransferase; AST, aspartate aminotransferase, TG, triglyceride; FFA, free fatty acid; FBG, fasting blood glucose.

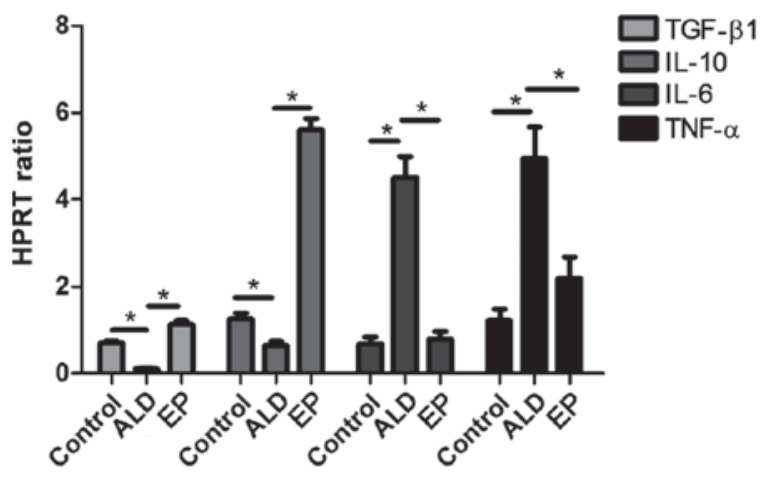

Liver tissue

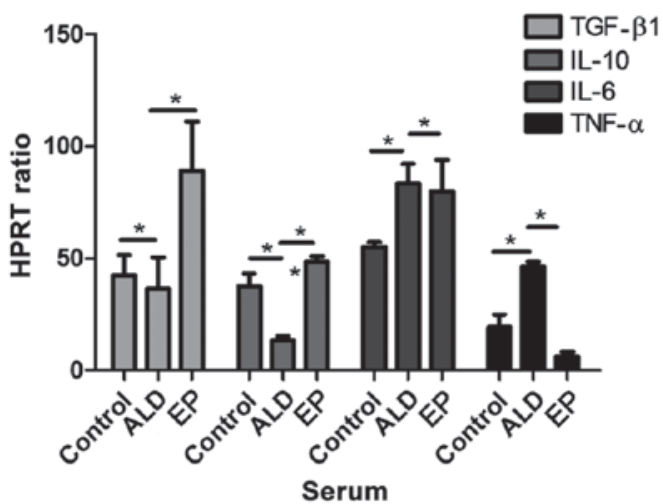

Figure 2. Comparisons of mRNA expression levels of inflammatory factors in serum and liver tissues of each experimental group (" $\mathrm{P}<0.05)$.

EP decreases the expression levels of pro-inflammatory factors and increased the expression levels of anti-inflammatory factors in $A L D$. As shown in Fig. 2, the mRNA expression levels of anti-inflammatory factors [transforming growth factor- $\beta$ (TGF- $\beta$ )/interleukin-10 (IL-10)] in liver tissues and serum in EP group were significantly increased compared with those in ALD group, and the differences were statistically significant. The mRNA expression levels of pro-inflammatory factors (IL-6/TNF- $\alpha$ ) in EP group were significantly decreased compared with those in ALD group, and the differences were statistically significant $(\mathrm{P}<0.05)$, proving that $\mathrm{EP}$ can alleviate the damage caused by ALD through increasing the expression levels of anti-inflammatory factors and decreasing the expression levels of pro-inflammatory factors.

$E P$ increased the MRNA expression level of PPAR- $\alpha$ and decreased the mRNA expression levels of CYP2E1, Nrf2 and $V L D L R$. As shown in Fig. 3, the mRNA expression levels of CYP2E1, Nrf2 and VLDLR in ALD group were significantly increased compared with those in EP group, and the differences were statistically significant $(\mathrm{P}<0.05)$. However, the mRNA expression level of PPAR- $\alpha$ in ALD group was significantly decreased compared with that in EP group, and the difference was statistically significant $(\mathrm{P}<0.05)$, indicating 

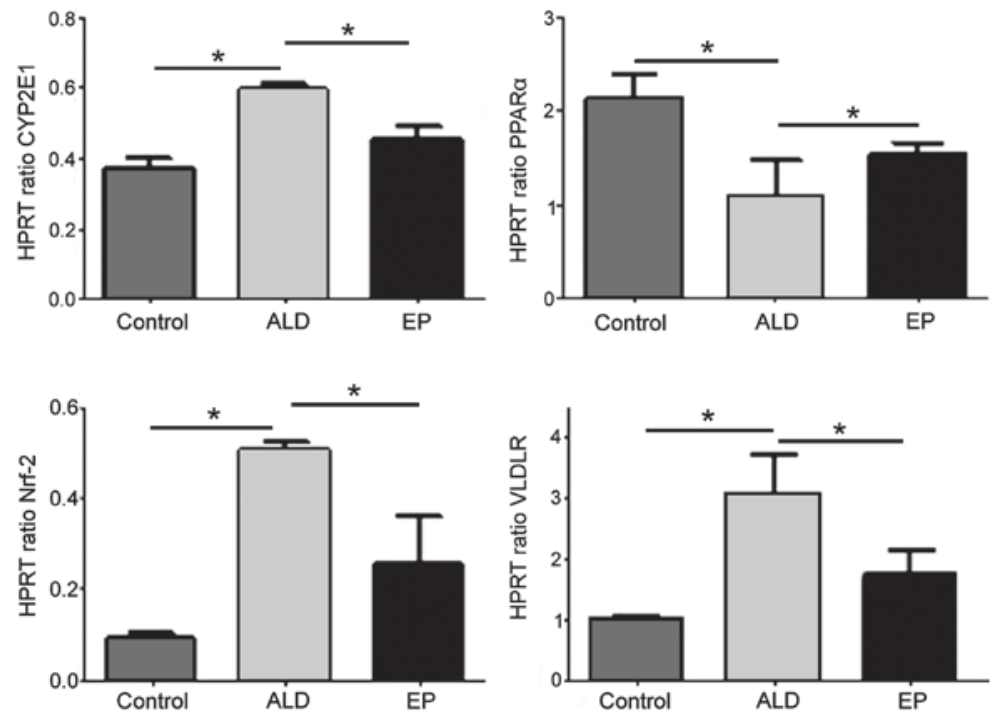

Figure 3. Comparisons of mRNA expression levels of PPAR- $\alpha$, cytochrome P4502E1 (CYP2E1), Nrf2 and very low density lipoprotein receptor (VLDLR) in liver tissues of each experimental group $(" \mathrm{P}<0.05)$. Nrf2, nuclear factor 2.
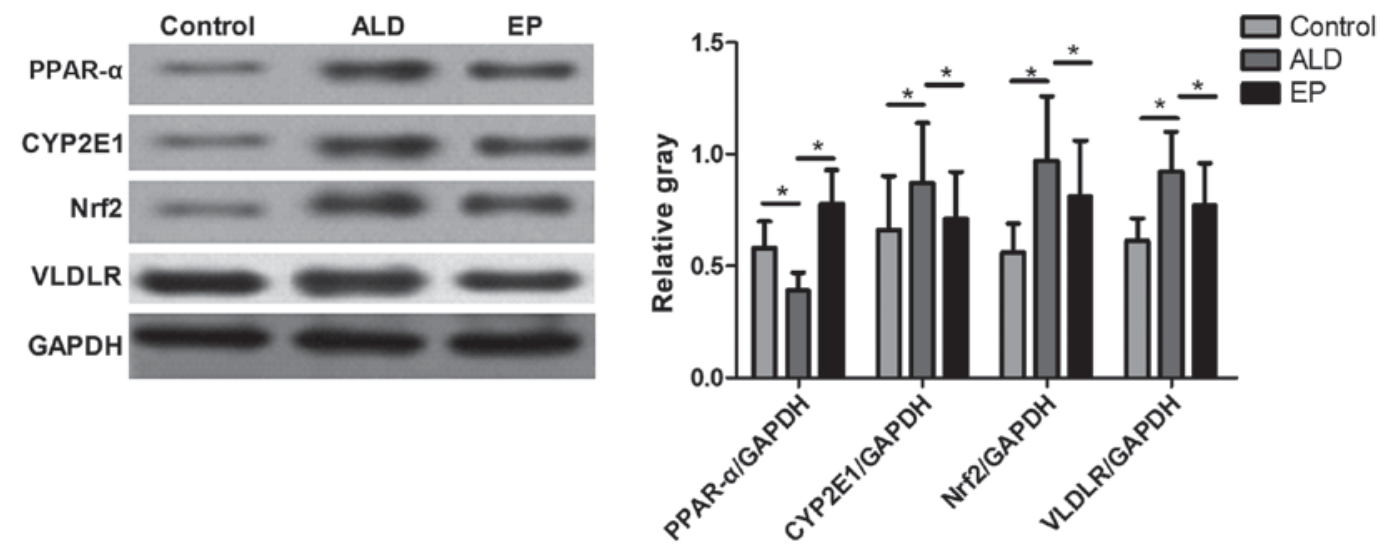

Figure 4. Comparisons of protein expression levels and the statistical analysis of the gray value of PPAR- $\alpha$, CYP2E1, Nrf2 and VLDLR in liver tissues in each experimental group. $(" \mathrm{P}<0.05)$. Nrf2, nuclear factor 2; VLDLR, very low density lipoprotein receptor.
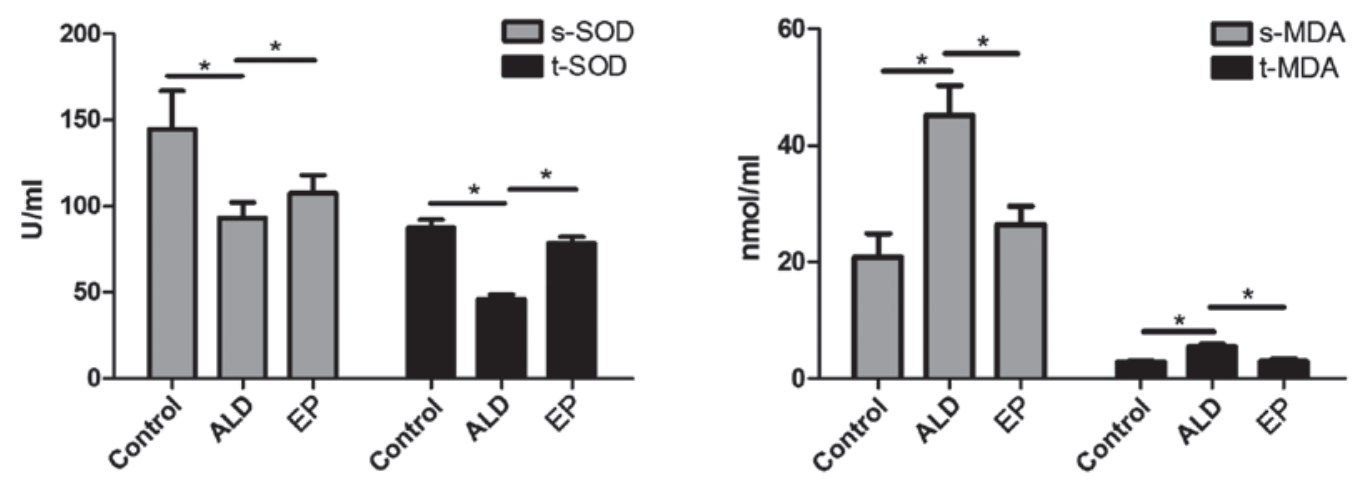

Figure 5. Comparison of SOD activity and MDA content in plasma and liver tissues of each experimental group ( $\mathrm{P}<0.05$ ). SOD, superoxide dismutase; MDA, malondialdehyde.

that EP can inhibit the expression levels of CYP2E1, Nrf2 and VLDLR and increase the expression of PPAR- $\alpha$ in ALD.

EP negatively regulated PPAR- $\alpha-C Y P 2 E 1$ signaling pathway and ROS-Nrf2 signaling pathway to reduce the VLDLR protein expression level. As shown in Fig. 4, the protein expression levels of CYP2E1, Nrf2 and VLDLR in ALD group were significantly increased compared with those in EP group, but the protein expression level of PPAR- $\alpha$ in ALD group was significantly decreased compared with that in EP group and the 
difference was statistically significant $(\mathrm{P}<0.05)$, indicating that EP can inhibit the protein expression levels of CYP2E1, Nrf2 and VLDLR and increase the protein expression of PPAR- $\alpha$ in ALD. As shown in Fig. 5, the SOD content in plasma and liver tissues in EP group was significantly increased compared with that in ALD group, and the difference was statistically significant $(\mathrm{P}<0.05)$; but the MDA content in EP group was significantly decreased compared with that in ALD group $(\mathrm{P}<0.05)$, indicating that EP can alleviate the oxidative stress injury caused by ALD.

As shown in Figs. 3-5, in EP group, CYP2E1 expression and MDA content were decreased, while PPAR- $\alpha$ expression and SOD content were increased, indicating that EP can negatively regulate the CYP2E1-PPAR- $\alpha$-ROS signaling pathway to alleviate the oxidative stress injury. However, in EP group, the expression levels of Nrf2 and VLDLR were decreased, indicating that EP can negatively regulate the Nrf2-VLDLR signaling pathway and reduce the VLDLR expression to alleviate the alcoholic fatty liver.

\section{Discussion}

The results of this study confirmed that EP can reduce the serum ALT/AST levels, TG and FFA concentrations and VLDLR protein expression in ALD mice, and alleviate the degeneration degree of alcoholic fatty liver, which has a good preventive and protective effect on ALD. Studies have confirmed that the oxidative stress, inflammation and lipid metabolism disorder caused by alcohol metabolism are important causes of ALD (1), so we investigated the preventive and protective effect of EP on ALD from the inflammation, oxidative stress and lipid metabolism.

Studies have confirmed that TNF- $\alpha$ can stimulate the decomposition of adipose tissues and increase the FFA content in the body (15), but the excessive FFA will be transported to the liver and accumulated in hepatocytes (16), eventually leading to the fatty degeneration or even necrosis of hepatocytes (17). Studies have also shown that the action of excessive FFA on hepatocytes can increase the oxygen consumption of liver tissues and aggravate the lipid peroxidation, further aggravating the liver injury (18). Therefore, it is speculated that EP can alleviate the alcoholic liver injury due to its strong anti-inflammatory effect, which can inhibit the expression levels of pro-inflammatory factors and increase the expressions of anti-inflammatory factors. In this experiment, compared with those in ALD group (Fig. 2), the mRNA expression levels of pro-inflammatory factors (IL-6/TNF- $\alpha$ ) in liver tissues and plasma in EP group were significantly decreased, while the mRNA expression levels of anti-inflammatory factors (TGF- $\beta / \mathrm{IL}-10)$ were significantly increased, which were consistent with our speculation.

In addition, it is reported in the literature (19) that SOD is one of the important antioxidant enzymes for the body to scavenge free radicals, and the intake of alcohol can inhibit its activity and accelerate liver injury (20). It is also reported in the literature that oxidative stress can induce the hepatocyte mitochondrial dysfunction, leading to hepatocyte degeneration or even necrosis (21). Therefore, it is speculated that the strong antioxidant effect of EP is one of the reasons to alleviate alcoholic liver injury, which is manifested in that it can increase the SOD activity and decrease the MDA production.
In this study, the SOD activities in serum and liver tissues in EP group (Fig. 5) were significantly increased compared with those in ALD group, but the MDA content was decreased, and the study proved that MDA is one of the important products of oxidative stress (22). The results of this experiment are also consistent with our speculation.

A further study (23) indicated that EP regulation of inflammation and oxidative stress is closely related to PPAR- $\alpha$. Studies (24) have confirmed that the phosphorylated protein PPAR- $\alpha$ can enhance the body's sensitivity to insulin, inhibit the production of inflammatory factors and inflammation and inhibit the oxidative stress response; and its decreased expression can aggravate the inflammation and oxidative stress injury (25). It has been reported in literature (26) that CYP2E1 can inhibit the expression of PPAR- $\alpha$. Therefore, it is speculated that EP can negatively regulate the CYP2E1-PPAR- $\alpha$ signaling pathway, thereby alleviating the oxidative stress and inflammatory injury. According to the experimental results (Figs. 3 and 4), the mRNA transcriptional level and protein expression level of CYP2E1 in EP group were significantly decreased compared with those in ALD group, but the mRNA transcriptional level and protein expression level of PPAR- $\alpha$ were significantly increased, which are consistent with our speculation.

It has been reported that oxidative stress can activate $\mathrm{Nrf} 2$, and the activation of Nrf2 can induce the VLDLR overexpression in hepatocytes, leading to alcoholic liver injury (6). Nrf2 gene silencing can inhibit the upregulation of VLDLR in hepatocytes caused by oxidative stress (27). Therefore, it is speculated that one of the mechanisms by which EP protects ALD is that EP can negatively regulate the Nrf2-VLDLR signaling pathway. The experimental results (Figs. 3 and 4) showed that EP could downregulate the mRNA and protein expression levels of Nrf2 and VLDLR in EP group, which is consistent with our speculation. In conclusion, we consider that the mechanism by which EP alleviates ALD involves its anti-inflammatory and anti-oxidative effects; EP can negatively regulate the CYP2E1PPAR- $\alpha$ signaling pathway to inhibit the oxidative stress response, thus negatively regulating the Nrf2-VLDLR signaling pathway to alleviate the severity of ALD.

In conclusion, the experimental results showed that EP can increase the levels of anti-inflammatory factors and decrease the levels of pro-inflammatory factors, enhance the SOD activity and decrease the FFA and TG contents through anti-inflammatory and anti-oxidative effects; moreover, it can upregulate the PPAR- $\alpha$ expression by negative regulation of CYP2E1-PPAR- $\alpha$ signaling pathway and downregulate the Nrf2 expression by negative regulation of Nrf2-VLDLR signaling pathway, thus alleviating ALD. This may be the new mechanism of the many plant ingredients alleviating ALD, which needs further experimental study. This finding, with important clinical significance, provides a new direction for the treatment and prevention of ALD.

\section{Acknowledgements}

Not applicable.

\section{Funding}

No funding was received. 


\section{Availability of data and materials}

All data generated or analyzed during this study are included in this published article.

\section{Authors' contributions}

FS designed the study, ZW was involved in the animal model establishment, WL and YL performed data analysis. FS was a major contributor in writing the manuscript. All authors read and approved the final manuscript.

\section{Ethics approval and consent to participate}

This study was approved by the Animal Ethics Committee of Institute of Materia Medica of Shandong Academy of Medical Sciences (Jinan, China).

\section{Consent for publication}

Not applicable.

\section{Competing interests}

The authors declare that they have no competing interests.

\section{References}

1. Yin HQ, Choi YJ, Kim YC, Sohn DH, Ryu SY and Lee BH: Salvia miltiorrhiza Bunge and its active component cryptotanshinone protects primary cultured rat hepatocytes from acute ethanol-induced cytotoxicity and fatty infiltration. Food Chem Toxicol 47: 98-103, 2009.

2. Cioboată R, Găman A, Traşcă D, Ungureanu A, Docea AO, Tomescu P, Gherghina F, Arsene AL, Badiu C, Tsatsakis AM, et al: Pharmacological management of non-alcoholic fatty liver disease: Atorvastatin versus pentoxifylline. Exp Ther Med 13: 2375-2381, 2017.

3. Tilg H, Moschen AR and Kaneider NC: Pathways of liver injury in alcoholic liver disease. J Hepatol 55: 1159-1161, 2011.

4. Zhong W, Zhao Y, Tang Y, Wei X, Shi X, Sun W, Sun X, Yin X, Sun X, Kim S, et al: Chronic alcohol exposure stimulates adipose tissue lipolysis in mice: Role of reverse triglyceride transport in the pathogenesis of alcoholic steatosis. Am J Pathol 180: 998-1007, 2012.

5. Gurel H, Genc H, Celebi G, Sertoglu E, Cicek AF, Kayadibi H, Ercin CN and Dogru T: Plasma pentraxin-3 is associated with endothelial dysfunction in non-alcoholic fatty liver disease. Eur Rev Med Pharmacol Sci 20: 4305-4312, 2016.

6. Wang Z, Dou X, Li S, Zhang X, Sun X, Zhou Z and Song Z: Nuclear factor (erythroid-derived 2)-like 2 activation-induced hepatic very-low-density lipoprotein receptor overexpression in response to oxidative stress contributes to alcoholic liver disease in mice. Hepatology 59: 1381-1392, 2014.

7. Jo H, Choe SS, Shin KC, Jang H, Lee JH, Seong JK, Back SH and Kim JB: Endoplasmic reticulum stress induces hepatic steatosis via increased expression of the hepatic very low-density lipoprotein receptor. Hepatology 57: 1366-1377, 2013.

8. Fawcett TW, Martindale JL, Guyton KZ, Hai T and Holbrook NJ: Complexes containing activating transcription factor (ATF)/cAMP-responsive-element-binding protein (CREB) interact with the CCAAT/enhancer-binding protein (C/EBP)-ATF composite site to regulate Gadd153 expression during the stress response. Biochem J 339: 135-141, 1999.

9. Harding HP, Zhang Y, Bertolotti A, Zeng H and Ron D: Perk is essential for translational regulation and cell survival during the unfolded protein response. Mol Cell 5: 897-904, 2000.
10. Lieber CS and DeCarli LM: Effects of mineral and vitamin supplementation on the alcohol-induced fatty liver and microsomal induction. Alcohol Clin Exp Res 13: 142-143, 1989.

11. Wang $X$ and Cederbaum AI: Acute ethanol pretreatment increases FAS-mediated liver injury in mice: Role of oxidative stress and CYP2E1-dependent and -independent pathways. Free Radic Biol Med 42: 971-984, 2007.

12. Kozutsumi Y, Segal M, Normington K, Gething MJ and Sambrook J: The presence of malfolded proteins in the endoplasmic reticulum signals the induction of glucose-regulated proteins. Nature 332: 462-464, 1988.

13. Zhang X, Wang Z, Li J, Gu D, Li S, Shen C and Song Z: Increased 4-hydroxynonenal formation contributes to obesity-related lipolytic activation in adipocytes. PLoS One 8: e70663, 2013.

14. Tsung A, Kaizu T, Nakao A, Shao L, Bucher B, Fink MP, Murase N and Geller DA: Ethyl pyruvate ameliorates liver ischemia-reperfusion injury by decreasing hepatic necrosis and apoptosis. Transplantation 79: 196-204, 2005.

15. Castro Cabezas M, Erkelens DW and van Dijk H: Free fatty acids: Mediators of insulin resistance and atherosclerosis. Ned Tijdschr Geneeskd 146: 103-109, 2002 (In Dutch).

16. Yellen P and Foster DA: Inhibition of fatty acid synthase induces pro-survival Akt and ERK signaling in K-Ras-driven cancer cells. Cancer Lett 353: 258-263, 2014.

17. Boden G, She P, Mozzoli M, Cheung P, Gumireddy K, Reddy P, Xiang X, Luo Z and Ruderman N: Free fatty acids produce insulin resistance and activate the proinflammatory nuclear factor-kappaB pathway in rat liver. Diabetes 54: 3458-3465, 2005.

18. Chang BH, Li L, Paul A, Taniguchi S, Nannegari V, Heird WC and Chan L: Protection against fatty liver but normal adipogenesis in mice lacking adipose differentiation-related protein. Mol Cell Biol 26: 1063-1076, 2006.

19. Samarghandian S, Azimi-Nezhad M, Borji A, Samini M and Farkhondeh T: Protective effects of carnosol against oxidative stress induced brain damage by chronic stress in rats. BMC Complement Altern Med 17: 249, 2017.

20. Collins AR: Oxidative DNA damage, antioxidants, and cancer. BioEssays 21: 238-246, 1999.

21. Xiong Y, Ye T, Wang M, Xia Y, Wang N, Song X, Wang F, Liu L, Zhu Y, Yang F, et al: A novel cinnamide YLT26 induces breast cancer cells apoptosis via ROS-mitochondrial apoptotic pathway in vitro and inhibits lung metastasis in vivo. Cell Physiol Biochem 34: 1863-1876, 2014.

22. Yang R, Han X, Delude RL and Fink MP: Ethyl pyruvate ameliorates acute alcohol-induced liver injury and inflammation in mice. J Lab Clin Med 142: 322-331, 2003.

23. Naour S, Espinoza BM, Aedo JE, Zuloaga R, Maldonado J, Bastias-Molina M, Silva H, Meneses C, Gallardo-Escarate C, Molina A, et al: Transcriptomic analysis of the hepatic response to stress in the red cusk-eel (Genypterus chilensis): Insights into lipid metabolism, oxidative stress and liver steatosis. PLoS One 12: e0176447, 2017.

24. Ramadori P, Drescher H, Erschfeld S, Schumacher F, Berger C, Fragoulis A, Schenkel J, Kensler TW, Wruck CJ, Trautwein C, et al: Hepatocyte-specific Keapl deletion reduces liver steatosis but not inflammation during non-alcoholic steatohepatitis development. Free Radic Biol Med 91: 114-126, 2016.

25. Sun X, Yamasaki M, Katsube T and Shiwaku K: Effects of quercetin derivatives from mulberry leaves: Improved gene expression related hepatic lipid and glucose metabolism in shortterm high-fat fed mice. Nutr Res Pract 9: 137-143, 2015.

26. Hamed AM, El-Kharashi OA, Boctor SS and Abd-Elaziz LF: Potential involvement of PPAR $\alpha$ activation in diminishing the hepatoprotective effect of fenofibrate in NAFLD: Accuracy of non-invasive panel in determining the stage of liver fibrosis in rats. Biomed Pharmacother 85: 68-78, 2017.

27. Qiu P, Dong Y, Li B, Kang XJ, Gu C, Zhu T, Luo YY, Pang MX, $\mathrm{Du} \mathrm{WF}$ and Ge WH: Dihydromyricetin modulates p62 and autophagy crosstalk with the Keap-1/Nrf2 pathway to alleviate ethanol-induced hepatic injury. Toxicol Lett 274: 31-41, 2017.

This work is licensed under a Creative Commons Attribution-NonCommercial-NoDerivatives 4.0 International (CC BY-NC-ND 4.0) License. 